\title{
Changes in views on digital intraoral scanners among dental hygienists after training in digital impression taking
}

\author{
Hye-Ran Park', Ji-Man Park², Youn-Sic Chun ${ }^{3}$, Kkot-Nim Lee $^{1}$ and Minji Kim ${ }^{3 *}$
}

\begin{abstract}
Backgrounds: Despite the rapid development of digital dentistry, the use of digital intraoral scanners remains limited. The aim of this study was to evaluate the changes in views on intraoral scanners among dental hygienists after training.

Methods: Thirty-four dental hygienists with $>3$ years of clinical experience participated and were divided into 2 groups : iTero and Trios groups. Participants of each group practiced the usage of both intraoral scanners, for total 12 times over 4 sessions, Questionnaires were given to participants at two different times; prior to and after the completion of the training sessions. The parameters of questionnaires included on difficulty of use, patient discomfort, awareness, preference, and clinical usefulness of intraoral scanners and comparison of two types of scanners.

Results: Upon the completion of the training, both iTero and Trios groups gave positive feedback on anticipated accuracy, efficiency, and clinical usefulness. More participants of the iTero group responded that the level of difficulty of use and patient discomfort was greater than Trios. Both groups preferred Trios for its clinical usefulness.

Conclusions: The perceptions of dental hygienists on usage of intraoral scanner and digital impression improved positively with the training. The participants favored Trios over iTero in terms of difficulty of use, patient comfort, and clinical usefulness. This study showed that appropriate training could change the views on the efficiency of intraoral scanners positively among dental hygienists.
\end{abstract}

Keywords: Digital intraoral scanner, Perception of digital impression, Training of intraoral scanner

\section{Backgrounds}

Computer-aided design/computer-aided manufacture (CAD/CAM) was first introduced in the field of dentistry in the 1980s; since then, the use of digital technology has been rapidly increased in dentistry, though having some arguments on its accuracy [1-4]. The conventional impression-taking method may pose patient discomfort and possibility of deformation which could be affected by the type of impression material [5-9], impression tray [10-12], and impression technique [13]. The digital impression technique could overcome these limitations by providing simple operating system $[2,14]$, accuracy, and improved patient comfort $[15,16]$. Additional advantages

\footnotetext{
* Correspondence: minjikim@ewha.ac.kr

${ }^{3}$ Department of Orthodontics, Graduate School of Clinical Dentistry, Ewha

Womans University, 1071, Anyangcheon-ro, Yangcheon-gu, Mokdong

Hospital, Seoul, Korea

Full list of author information is available at the end of the article
}

include providing a preview of three-dimensional (3D) images while taking the impression. Some studies evaluated on accuracy of digital impression technique using intraoral scanners and reported that accuracy could be affected by materials or scanning strategies [2, 4]. Previous studies on the digital impression-taking using intraoral scanners have been limited to the accuracy and efficiency of intraoral scanners [17-19]. Some of previous studies compared the inconvenience and difficulty of digital impression method using between intraoral scanners compared to conventional impression methods among dental students in the university $[15,20,21]$. The aim of this study is to evaluate the changes in views on intraoral scanners among dental hygienists after training in digital impression.

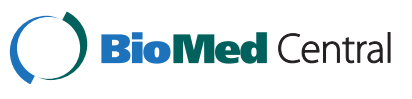

(c) 2015 Park et al. Open Access This article is distributed under the terms of the Creative Commons Attribution 4.0 International License (http://creativecommons.org/licenses/by/4.0/), which permits unrestricted use, distribution, and reproduction in any medium, provided you give appropriate credit to the original author(s) and the source, provide a link to the Creative Commons license, and indicate if changes were made. The Creative Commons Public Domain Dedication waiver (http://creativecommons.org/publicdomain/zero/1.0/) applies to the data made available in this article, unless otherwise stated. 


\section{Methods}

\section{Participants}

Thirty-four dental hygienists with a clinical experience of at least 3 years who had no experience in using intraoral scanners participated in the study and they were divided into 2 groups; an iTero $(\mathrm{n}=17)$ and a Trios $(\mathrm{n}=17)$ group using random allocation method. All participants were recruited voluntarily after the informative session about the study and written consent forms were obtained. This study was approved by the Institutional Review Board Committee of Ewha Womans University Medical College (Approval number: ECT14-02A-27).

\section{Intraoral scanners}

In this study, 2 different types of 3D intraoral scanners, iTero $^{\circ}$ (Align Technology Inc. Santa Clara, California) and Trios $^{\oplus}$ (3Shape dental systems, Copenhagen, Denmark), were used. The iTero is operated by the parallel confocal principle and acquires 3D data by over 100,000 red laser beams to the object and fusing the acquired images.The weight of the wand is $1,100 \mathrm{~g}$. The Trios is operated by the confocal principle with the video-recording method based on the real-time rendering technique. The scanner head of Trios weighs $760 \mathrm{~g}$. Both intraoral scanners were operated according to the manufacturer's instructions.

\section{Study design and Workflow}

All participants not only performed as operators, but also underwent to have scanning experience as patients. The participants were fully trained with only one scanner according to assigned group, and the other scanner was used once for the comparing purpose between two different scanners. On the first day, each group of participants filled out the first questionnaire before the training was initiated. Then, the principles and operational concepts of iTero and Trios intraoral scanner devices were introduced. Practice session of intraoral scanner on models was followed by actual intraoral scanning activity. Digital impression from actual intraoral scan was obtained twice. On the second day, each group of participants practiced and took intraoral digital impression for three times. And all participants also underwent for iTero scanning experience as patients, which was operated by a qualified professional. On the third day, all participants again acquired actual intraoral images for 3 times. And they underwent Trios scanning experience as patients, which was operated by a qualified professional. On the last day, all participants acquired actual intraoral digital impression for twice and then practiced once on models again. Finally, each group of participants exchanged to the other scanner and acquired digital impression once intraorally. Upon the completion of the training sessions, all participants completed the second questionnaire.(Fig. 1)

\section{Questionnaire configuration}

Two questionnaires were administered during the study (Additional file 1). The first preliminary questionnaire was given initially before the training sessions and the second questionnaire was completed upon the completion of all the training sessions. The first questionnaire was administered before the training sessions and evaluated three

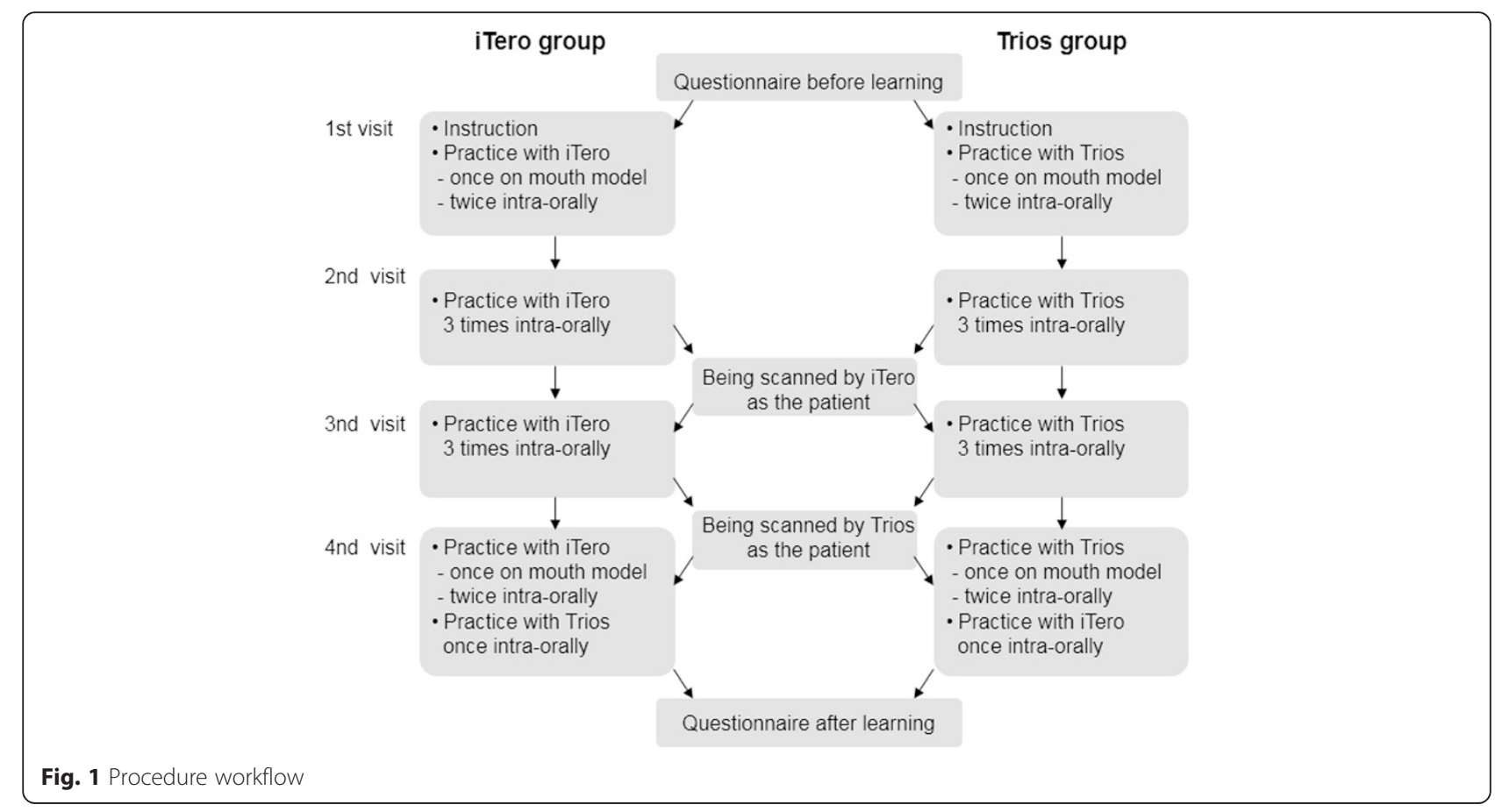


main parameters: difficulties of using intraoral scanners with digital impression method compared to conventional impression-taking method, patient comfort, and degree of awareness about intraoral scanners. The parameter of awareness included anticipated accuracy, patient convenience, efficiency, clinical application, and interest in further use. The second questionnaire was administered upon the completion of all the training sessions and evaluated all the above parameters in addition to parameters of preference, clinical usefulness, and comparison of the two different types of scanners.

\section{Statistical analysis}

All statistical analyses were performed using IBM Statics 19.0 (SPSS Inc., Chicago, USA) software. The paired ttest was used to test differences. The level of significance was set at 0.05 .

\section{Results}

Views on difficulty of use and patient comfort before and after training

There was no significant difference in views on difficulties of using intraoral scanner devices for digital impression-taking method compared to that with conventional impression-taking methods before and after training in both the iTero and Trios groups (Table 1). A similar result was shown for parameter of patient comfort.

In the iTero group, prior to the training sessions, $53 \%$ of participants responded that digital impression taking method may be easier than taking conventional impression using rubber materials. However, after the training sessions, only $24 \%$ of participants in iTero group responded that digital impression taking method may be easier compared to conventional rubber impression taking method (Fig. 2). In the Trios group, the response rate for the same question was $47 \%$, prior to and after the training. Furthermore, when the difficulties of using digital impression taking method was compared to the conventional impression taking method using alginate material the participants responded digital impression to be more difficult as $71 \%$ and $82 \%$ before and after training, respectively, in the iTero group, and $65 \%$ and $53 \%$ before and after training, respectively, in the Trios group.

To evaluate parameter of patient comfort, all the participants underwent digital impression scanning experience using iTero and Trios. Before the training, $65 \%$ of participants in the iTero group and $41 \%$ of participants in the Trios group responded that digital impression taking may be more comfortable to patients than impression taking using rubber materials; these rates decreased to $53 \%$ in the iTero group and increased to $47 \%$ in the Trios group. In comparison with patient comfort during impression taking using alginate, subjects who answered that digital impression taking was more convenient decreased from $53 \%$ to $41 \%$ in the iTero group and remained the same in the Trios group (53\%).

The number of subjects who responded that digital impression taking was easier than conventional impression taking as an operator was greater for rubber impression materials than for alginate. This was due to difficulty, and higher level of expected precision in the use of rubber materials. The number of responses that digital impression taking was more difficult generally increased after training in the iTero group and decreased in the Trios group. With regard to patient comfort, the number of responses that digital impression taking was more convenient was almost similar before and after training in both groups.

\section{Awareness about digital impression taking before and after training}

As shown in Table 2, scores for the anticipated accuracy of digital impression taking method increased after the training in the iTero group. With regard to clinical usefulness, after the training sessions, more participants in the iTero group answered that digital scanners could be helpful tools in dental clinics. However, there were no significant differences for other parameters before and after training in the iTero group. In the Trios group, there were no significant differences for any parameters.

The participants from both groups generally showed positive responses for all parameters of digital impression taking method (Fig. 3).

Table 1 Difficulty of use and patient discomfort while using intraoral scanners and rubber and alginate materials, before and after training (10-point Likert scale)

\begin{tabular}{|c|c|c|c|c|c|c|c|}
\hline \multirow[t]{3}{*}{ Variables } & \multirow{3}{*}{$\begin{array}{l}\text { Conventional } \\
\text { impression material }\end{array}$} & \multicolumn{3}{|c|}{ iTero group } & \multicolumn{3}{|l|}{ Trios group } \\
\hline & & \multicolumn{2}{|l|}{ Mean (SD) } & \multirow[t]{2}{*}{$p$-value } & \multicolumn{2}{|l|}{ Mean (SD) } & \multirow[t]{2}{*}{$p$-value } \\
\hline & & Before & After & & Before & After & \\
\hline \multirow[t]{2}{*}{ Level of difficulty } & Rubber & $4.65(2.09)$ & $5.65(1.94)$ & .101 & $5.12(1.80)$ & $5.00(2.26)$ & .847 \\
\hline & Alginate & $6.18(2.38)$ & $7.29(1.65)$ & .128 & $5.77(2.46)$ & $5.59(2.62)$ & .779 \\
\hline \multirow[t]{2}{*}{ Level of Patient discomfort } & Rubber & $4.29(2.05)$ & $4.12(1.83)$ & .726 & $4.47(2.63)$ & $4.47(2.79)$ & 1.000 \\
\hline & Alginate & $4.88(2.29)$ & 5.18 (2.33) & .663 & $4.24(2.10)$ & $4.47(2.76)$ & .680 \\
\hline
\end{tabular}

10-point Likert scale: $0 \sim 4$ = low score (Digital impression taking was easier and more convenient) 5; neutral; $6 \sim 10=$ high score (Digital impression taking was difficult and inconvenient) 


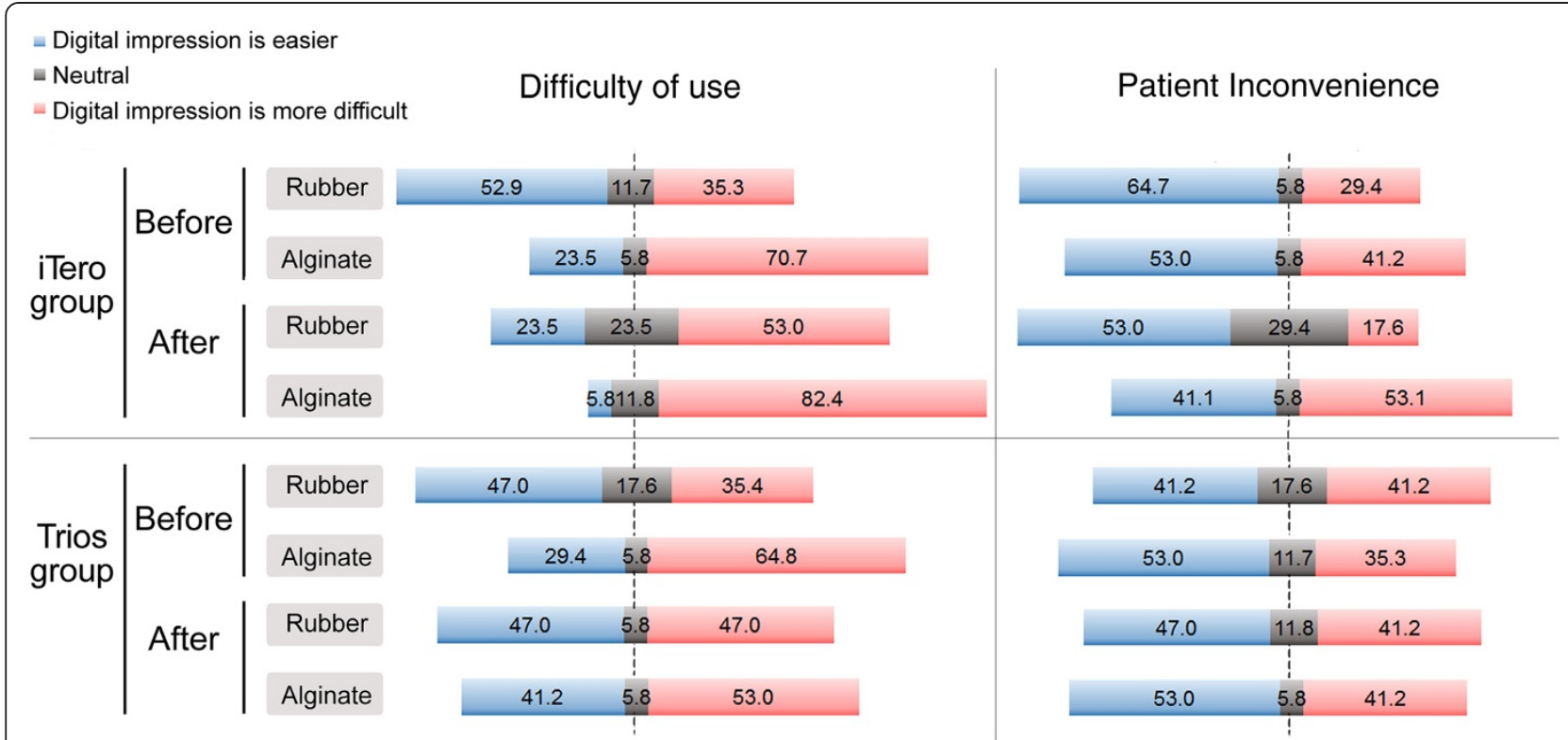

Fig. 2 Difficulty of use and patient inconvenience between intraoral scanners and rubber and alginate materials before and after training (10-point Likert scale)

\section{Preference for digital impression taking}

As shown in Fig. 4, all participants from both groups gave a positive overall feedbacks for digital impression taking method. The results showed that $82.4 \%$ participants of both iTero and Trios groups showed willingness to use the intraoral scanner in the future. Participants agreed that the training for the intraoral scanner is useful; $94.1 \%$ participants in the iTero group and $88.2 \%$ participants in the Trios group. All subjects were interested in receiving information about intraoral scanners.

Views on clinical usefulness in the iTero and Trios groups In terms of the subjective weight of the scanner head between two intraoral scanners, in the iTero group, the

Table 2 Awareness about digital impression taking before and after training (7-point Likert scale)

\begin{tabular}{|c|c|c|c|c|c|c|c|}
\hline \multirow[t]{3}{*}{ Parameters } & \multirow{3}{*}{$\begin{array}{l}\text { Variables (Scores for agreement regarding } \\
\text { digital impression taking) }\end{array}$} & \multicolumn{3}{|l|}{ iTero } & \multicolumn{3}{|l|}{ Trios } \\
\hline & & \multicolumn{2}{|l|}{ Mean (SD) } & \multirow[t]{2}{*}{$p$-value } & \multicolumn{2}{|l|}{ Mean (SD) } & \multirow[t]{2}{*}{$p$-value } \\
\hline & & Before & After & & Before & After & \\
\hline \multirow[t]{2}{*}{ Accuracy } & More accurate than rubber impression taking & $4.29(1.45)$ & $4.82(1.24)$ & $0.024^{*}$ & $5.06(1.09)$ & $5.59(1.23)$ & 0.132 \\
\hline & More accurate than alginate impression taking & $4.77(1.60)$ & $5.35(1.62)$ & 0.096 & $5.47(1.13)$ & $6.06(1.09)$ & 0.086 \\
\hline Convenience & More efficient management of impression model & $5.88(1.69)$ & $6.29(1.49)$ & 0.130 & $6.41(0.80)$ & $6.59(0.62)$ & 0.484 \\
\hline \multirow[t]{4}{*}{ Efficiency } & Possibility of saving time compared to rubber impressions & $5.24(1.60)$ & $4.88(1.57)$ & 0.455 & $4.65(1.54)$ & $4.82(1.63)$ & 0.661 \\
\hline & Possibility of saving time compared to alginate impressions & $4.29(2.14)$ & $4.06(2.05)$ & 0.702 & $4.29(1.72)$ & $4.06(1.78)$ & 0.632 \\
\hline & Influence on simplification of the entire treatment process & $5.24(1.03)$ & $5.06(1.56)$ & 0.627 & $5.18(1.01)$ & $5.47(1.06)$ & 0.311 \\
\hline & Usefulness in attracting patient's attention. & $5.82(1.43)$ & $6.12(0.78)$ & 0.385 & $5.47(1.18)$ & $6.00(0.94)$ & 0.083 \\
\hline \multirow[t]{2}{*}{ Clinical usefulness } & Influence on increasing patient's trust. & $5.35(1.58)$ & $5.88(0.78)$ & 0.120 & $5.59(1.06)$ & $5.77(0.90)$ & 0.616 \\
\hline & Influence on promoting the dental clinic. & $5.35(1.50)$ & $5.88(0.99)$ & $0.034^{*}$ & $5.59(1.06)$ & $6.00(0.87)$ & 0.069 \\
\hline \multirow[t]{2}{*}{ Skill acquisition } & Ease of training in a short time & $4.47(1.28)$ & $4.06(1.30)$ & 0.370 & $4.65(1.46)$ & $4.65(1.73)$ & 1.000 \\
\hline & $\begin{array}{l}\text { Effect of proficiency in using digital impression techniques } \\
\text { on the accuracy }\end{array}$ & $6.24(0.83)$ & $6.59(0.71)$ & 0.251 & $6.18(0.88)$ & $6.18(0.72)$ & 1.000 \\
\hline \multicolumn{2}{|c|}{ Superior ability in taking digital impressions compared to other colleagues } & $5.18(1.33)$ & $5.41(1.27)$ & 0.299 & $5.65(1.00)$ & $5.71(0.85)$ & 0.817 \\
\hline \multicolumn{2}{|c|}{ Usefulness of digital impression in the clinical environment } & $4.82(1.28)$ & $5.18(1.29)$ & 0.370 & $5.47(0.80)$ & $5.53(1.23)$ & 0.848 \\
\hline \multicolumn{2}{|c|}{ Positive interest in taking digital impressions } & $5.59(1.06)$ & $5.29(0.92)$ & 0.206 & $5.65(0.79)$ & $5.71(1.11)$ & 0.848 \\
\hline
\end{tabular}

7 -point Likert scale: $1=$ very strongly disagree, $2=$ strongly disagree, $3=$ disagree, $4=$ neutral, $5=$ agree, $6=$ strongly agree, $7=$ very strongly agree, $*: p<0.05$ 


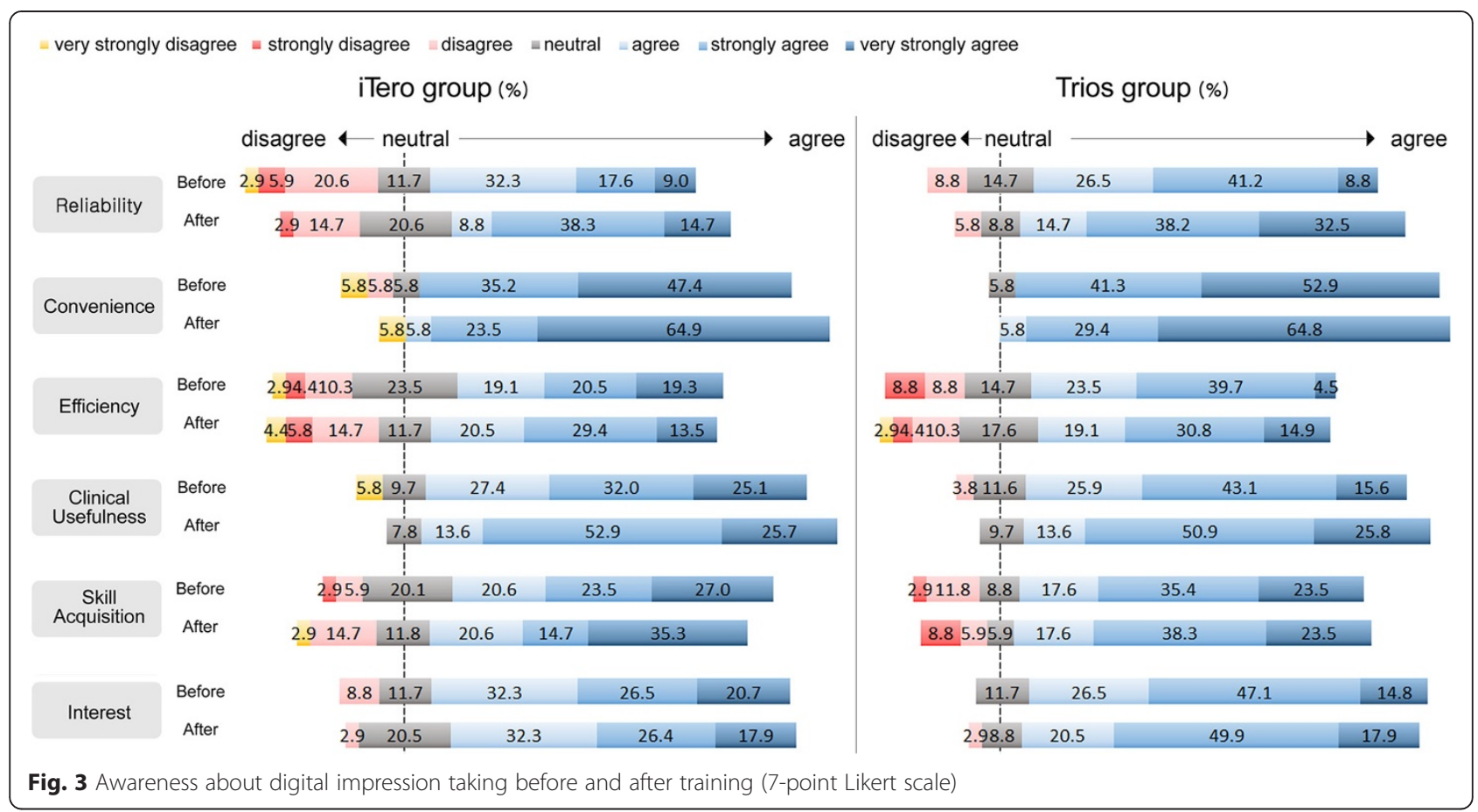

degree of agreement to the fact that the scanner head was light was significantly higher for Trios $(2.65 \pm 0.86)$ than for iTero $(1.65 \pm 0.78)$ (Table 3). Similar results were shown in Trios group. In the iTero group, the response that handling the scanner was convenient showed a significantly different degree of agreement for iTero (2.52 \pm 0.87$)$ and Trios (3.17 \pm 0.52 ). With regard to the ease of software and hardware operation in the Trios group, more respondents agreed that Trios was easy to operate.
These results suggested that positive responses for clinical usefulness were greater with Trios than for iTero intraoral scanner.

\section{Views on difficulty of use and patient comfort in the iTero and Trios groups}

As shown as Table 4, in the iTero group, the level of difficulty of use was rated higher for iTero (5.47 \pm 2.37) than for Trios $(4.23 \pm 1.92)$, as was the level of

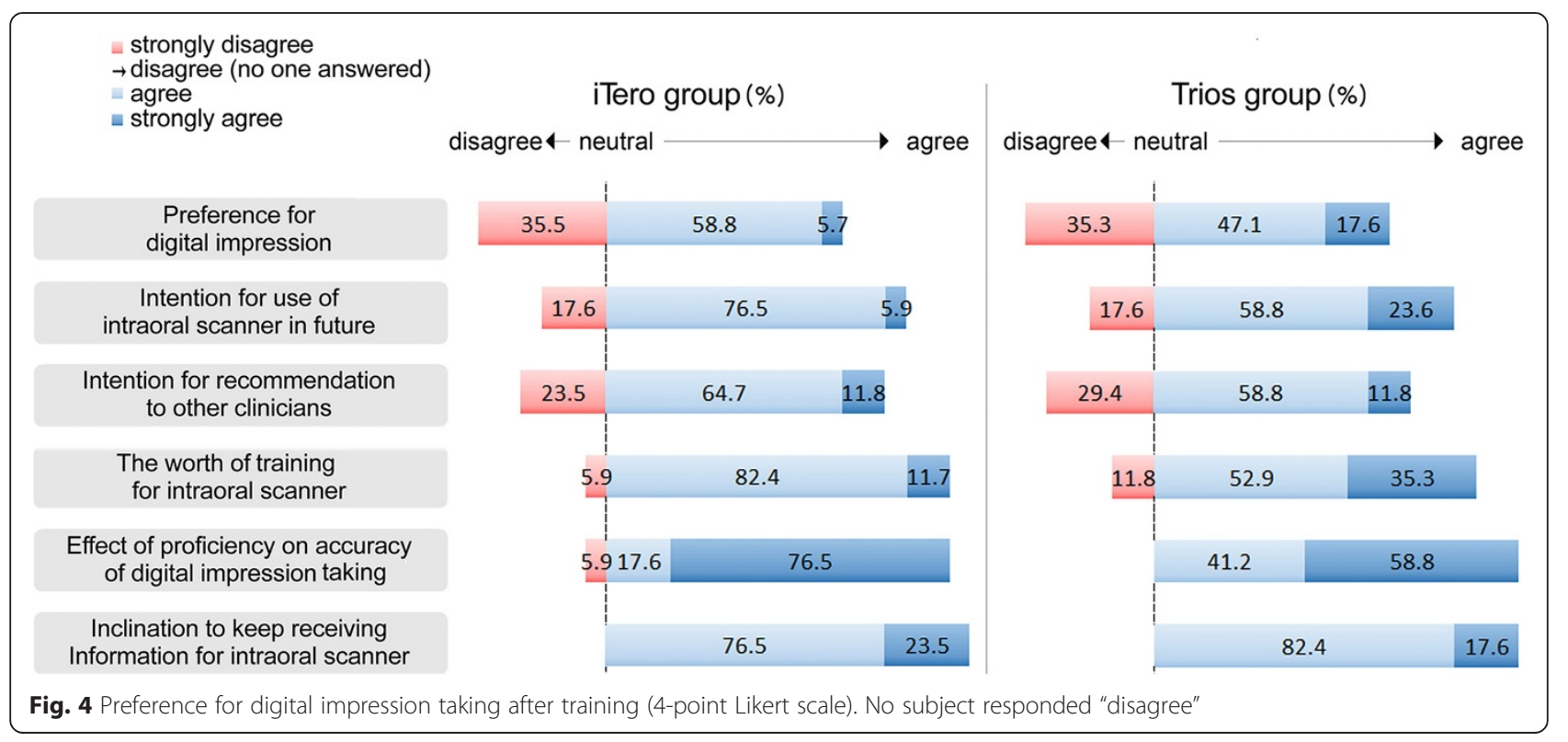


Table 3 Views on the clinical usefulness of iTero and Trios after training (4-point Likert scale)

\begin{tabular}{|c|c|c|c|c|c|c|}
\hline \multirow[t]{3}{*}{ Variables } & \multicolumn{3}{|l|}{ iTero group } & \multicolumn{3}{|l|}{ Trios group } \\
\hline & iTero & Trios & $p$-value & iTero & Trios & $p$-value \\
\hline & \multicolumn{3}{|l|}{ Mean (SD) } & \multicolumn{3}{|l|}{ Mean (SD) } \\
\hline Light weight head & $1.65(0.78)$ & $2.65(0.86)$ & $.000^{*}$ & $1.89(0.60)$ & $2.30(0.58)$ & $.014^{*}$ \\
\hline Small-sized head & $2.12(0.69)$ & $2.42(0.87)$ & .096 & $2.00(0.70)$ & $2.18(0.63)$ & .332 \\
\hline Easy to operate software & $2.89(0.85)$ & $2.95(0.82)$ & .750 & $2.24(0.75)$ & $3.00(0.50)$ & $.008^{*}$ \\
\hline Easy to operate hardware & $2.47(0.71)$ & $2.82(0.63)$ & .188 & $2.29(0.46)$ & $2.70(0.46)$ & $.004^{*}$ \\
\hline Good grip & $2.05(0.65)$ & $2.76(0.66)$ & $.009^{*}$ & $2.35(0.78)$ & $2.23(0.66)$ & .651 \\
\hline Quick training & $2.23(0.56)$ & $2.70(0.77)$ & .056 & $2.41(0.50)$ & $2.58(0.50)$ & .269 \\
\hline Comfortable handling & $2.52(0.87)$ & $3.17(0.52)$ & $.007^{*}$ & $2.58(0.71)$ & $2.70(0.77)$ & .579 \\
\hline
\end{tabular}

4-point Likert scale: $1=$ strongly disagree, $2=$ disagree, $3=$ agree, $4=$ strongly agree, ${ }^{*}: p<0.05$

patient comfort $(4.11 \pm 2.08,3.11 \pm 1.56$, respectively) . However, in the Trios group, the levels of difficulty and discomfort showed no significant difference between Trios and iTero.

\section{Discussion}

The clinical application of digital impression method has been increased continuously due to some advantages; possibility of immediate fabrication of intraoral models [22], and no requirement of impression trays and materials $[14,16]$. Previous study by Lee et al. [21] evaluated the efficiency, difficulty and participants' preference of a digital impression compared with a conventional impression methods. The result of the study reported that $40 \%$ participants of clinicians group agreed to the efficiency of digital impression taking while $53 \%$ of disagreed. And $33 \%$ of clinicians preferred digital impression taking, $37 \%$ favored conventional impression taking, and $30 \%$ had no preference. In addition, participants of dental students group perceived that digital impression was easier than conventional impression whereas the clinicians perceived that there was no difference between digital and conventional impression-taking methods. Yuzbasioglu et al. [15] reported that $100 \%$ of participants preferred digital impression-taking method using CEREC compared to conventional impression-taking methods in the parameters of following: patient comfort, sensitivity, gag reflex and user friendliness.

In the present study, although the participants practiced using the intraoral scanners only 12 times, the difficulty of use was generally similar between intraoral scanners and rubber or alginate impression materials. However, a greater number of participants responded that conventional alginate impression taking was easier than digital impression taking in both the iTero and Trios groups before and after training. This could have resulted from less time required for training and participants' familiarity with alginate impression material and technique. When digital impression-taking method was compared to conventional impression taking method with rubber materials, participants of both itero and Trios groups responded that intraoral scanners were more convenient. With regard to conventional impression taking with alginate material, participants in Trios group generally agreed that intraoral scanners were more convenient than alginate before and after training. These results were consistent with results of previous study [15]. Interestingly, the proportion of patients in the iTero group who answered that alginate impression taking was more comfortable than digital impression taking decreased from $64.8 \%$ to $53 \%$.

This study showed that $58.9 \%$ participants agreed to the efficiency of digital impression taking before training, and this percentage increased to $63.4 \%$ after training. Furthermore, the participants gave positive feedbacks for anticipated accuracy, patient comfort, clinical usefulness, ease of skill acquisition, and interest in further use. In this study, difficulty of use and patient discomfort were lower with Trios than with iTero. Moreover, as shown in Table 3, many participants responded that the head of

Table 4 Views on difficulty of use and patient discomfort while using iTero and Trios (10-point Likert scale)

\begin{tabular}{|c|c|c|c|c|c|c|}
\hline \multirow{3}{*}{ Variables } & \multicolumn{3}{|l|}{ iTero group } & \multicolumn{3}{|l|}{ Trios group } \\
\hline & iTero & Trios & $p$-value & iTero & Trios & $p$-value \\
\hline & \multicolumn{3}{|l|}{ Mean (SD) } & \multicolumn{3}{|l|}{ Mean (SD) } \\
\hline Level of difficulty & $5.47(2.37)$ & $4.23(1.92)$ & $.034^{*}$ & $5.70(2.08)$ & $4.52(2.26)$ & .061 \\
\hline Level of patient discomfort & $4.11(2.08)$ & $3.11(1.56)$ & $.007^{*}$ & $4.70(2.08)$ & $4.11(2.17)$ & .276 \\
\hline
\end{tabular}

10-point Likert scale: $0 \sim 4$; low score, easy and more convenient, 5; neutral, $6 \sim 10$; high score, difficult and inconvenient, ${ }^{*}: p<0.05$ 
the iTero intraoral scanner was heavier compared to Trios. However, the software of Trios scanner system was easier to operate than iTero system. The difference in scanner weight between iTero and Trios was $340 \mathrm{~g}$ (iTero, 1,100 g; Trios, $760 \mathrm{~g}$ ). In this study, the rate of positive response to questions investigating preference for digital impression taking was significant in the both iTero and Trios groups, suggesting the possibility of rapid digitalization in the field of dentistry. This study had a small sample size and a short duration for the training session, therefore, further studies should be conducted with larger sample size and prolonged period of training

\section{Conclusions}

This study evaluated progressive change in perceptions of digital intraoral scanners with short period of training in digital impression method among dental hygienists. The parameters of evaluation included difficulty of use, patient comfort, preference, and clinical usefulness.

Within the limitations of this study, training in the use of intraoral scanners has changed the views of dental hygienists positively. And the result of this study indicated that participants generally preferred Trios intraoral scanner over iTero as a operator. The usefulness of intraoral scanner could be a successful alternative to conventional impression-taking with proper training and increased clinical experiences in digital impression method.

\section{Additional file}

Additional file 1: Questionnaire. (PDF $300 \mathrm{~kb}$ )

\section{Competing interests}

The authors declare that they have no competing interests.

\section{Authors' contributions}

HRP carried out the survey and participated in training the subjects. JMP conceived the study and participated in the design of the study. YSC participated in coordinating the study and helped to draft the manuscript. KNL helped to draft and revise the manuscript. MK conceived and designed the study, helped to draft and revise the manuscript. All authors read and approved the final manuscript.

\section{Acknowledgements}

This study was supported by the Ewha Womans University Research Grant of 2013.

\footnotetext{
Author details

${ }^{1}$ Graduate School of Clinical Dentistry, Ewha Womans University, Seoul, Korea. ${ }^{2}$ Department of Prosthodontics and Dental Research Institute, Seoul National University Gwanak Dental Hospital, Seoul, Korea. ${ }^{3}$ Department of Orthodontics, Graduate School of Clinical Dentistry, Ewha Womans University, 1071, Anyangcheon-ro, Yangcheon-gu, Mokdong Hospital, Seoul, Korea.
}

Received: 30 December 2014 Accepted: 23 November 2015

Published online: 27 November 2015

\section{References}

1. Nedelcu RG, Persson AS. Scanning accuracy and precision in 4 intraoral scanners: an in vitro comparison based on 3-dimensional analysis. J Prosthet Dent. 2014;112(6):1461-71.

2. Ender A, Mehl A. Influence of scanning strategies on the accuracy of digital intraoral scanning systems. Int J Comput Dent. 2013;16(1):11-21.

3. Anh JW, Park JM, Chun YS, Kim MA, Kim MJ. A comparison on the precision of three-dimensional images acquired by two different digital intraoral scanners: Effects of tooth irregularity and scanning direction. Korean J Orthod 2015, In press.

4. Patzelt SB, Emmanouilidi A, Stampf S, Strub JR, Att W. Accuracy of full-arch scans using intraoral scanners. Clin Oral Investig. 2014;18(6):1687-94.

5. Keating AP, Knox J, Bibb R, Zhurov Al. A comparison of plaster, digital and reconstructed study model accuracy. J Orthod. 2008;35(3):191-201.

6. Lee H, Ercoli C, Funkenbusch PD, Feng C. Effect of subgingival depth of implant placement on the dimensional accuracy of the implant impression: an in vitro study. J Prosthet Dent. 2008;99(2):107-13.

7. Walker MP, Ries D, Borello B. Implant cast accuracy as a function of impression techniques and impression material viscosity. Int J Oral Maxillofac Implants. 2008;23:669-74.

8. Lee H, So JS, Hochstedler JL, Ercoli C. The accuracy of implant impressions: a systematic review. J Prosthet Dent. 2008;100(4):285-91.

9. Wee AG. Comparison of impression materials for direct multi-implant impressions. J Prosthet Dent. 2000;83(3):323-31.

10. Brosky ME, Pesun IJ, Lowder PD, Delong R, Hodges JS. Laser digitization of casts to determine the effect of tray selection and cast formation technique on accuracy. J Prosthet Dent. 2002;87(2):204-9.

11. Burns J, Palmer R, Howe L, Wilson R. Accuracy of open tray implant impressions: an in vitro comparison of stock versus custom trays. J Prosthet Dent. 2003;89(3):250-5.

12. Ceyhan JA, Johnson GH, Lepe X. The effect of tray selection, viscosity of impression material, and sequence of pour on the accuracy of dies made from dual-arch impressions. J Prosthet Dent. 2003:90(2):143-9.

13. Vigolo P, Fonzi F, Majzoub Z, Cordioli G. An evaluation of impression techniques for multiple internal connection implant prostheses. J Prosthet Dent. 2004;92(5):470-6.

14. Christensen GJ. Impressions are changing: deciding on conventional, digital or digital plus in-office milling. J Am Dent Assoc. 2009;140(10):1301-4.

15. Yuzbasioglu E, Kurt H, Turunc R, Bilir H. Comparison of digital and conventional impression techniques: evaluation of patients' perception, treatment comfort, effectiveness and clinical outcomes. BMC Oral Health 2014;14:10-6.

16. Wismeijer D, Mans R, van Genuchten M, Reijers HA. Patients' preferences when comparing analogue implant impressions using a polyether impression material versus digital impressions (Intraoral Scan) of dental implants. Clin Oral Implants Res. 2013;25(7):1-6.

17. Herbst D, Nel JC, Driessen CH, Becker PJ. Evaluation of impression accuracy for osseointegrated implant supported superstructures. J Prosthet Dent. 2000;83(5):555-61.

18. Choi HS, Kim SH. The Application of CAD/CAM in Dentistry. J Kor Dent Assoc. 2012;50:110-7.

19. Miyazaki T, Hotta Y, Kunii J, Kuriyama S, Tamaki Y. A review of dental CAD/CAM: current status and future perspectives from 20 years of experience. Dent Mater J. 2009;28(1):44-56.

20. Lee SJ, Gallucci GO. Digital vs. conventional implant impressions: efficiency outcomes. Clin Oral Implants Res. 2013;24(1):111-5.

21. Lee SJ, Macarthur RX, Gallucci GO. An evaluation of student and clinician perception of digital and conventional implant impressions. J Prosthet Dent. 2013;110(5):420-3.

22. Kugel G. Impression-taking: conventional methods remain steadfast as digital technology progresses. Compend Contin Educ Dent. 2014;35(3):202-3. 